patients for 3 domains (TWPI", absenteeism, presenteeism), and in PsA patients for 2 domains (absenteeism, activity impairment).

Conclusions: Golimumab s. c. 1 x monthly is an effective treatment in patients with RA, AS and PsA.

All scores of the WPAI showed a significant $(p<0.05)$ reduction in mean score values in each indication.

Golimumab leads to an improvement of work productivity and daily activities in all patients already within the first 3 months of treatment and provided sustained improvement in WPAI in patients with RA, PsA and AS.

Disclosure of Interest: I. Klaudius Employee of: MSD Sharp \& Dohme GmbH, K. Krueger: None declared, S. Remstedt: None declared, A. Thiele: None declared

DOI: 10.1136/annrheumdis-2018-eular.2925

\section{SAT0188 1 TOCILIZUMAB IN EARLY RHEUMATOID ARTHRITIS DELIVERS CLINICAL AND ULTRASOUND-CONFIRMED RAPID AND DEEP REMISSION WITH ABOLITION OF PD - TREMERA STUDY}

L. Hunt ${ }^{1}$, E. Hensor ${ }^{1,2}$, K. Naraghi ${ }^{1}$, R. J. Wakefield ${ }^{1,2}$, P. Emery ${ }^{1,2}$, M. H. Buch ${ }^{1,2}$. ${ }^{1}$ Leeds Institute of Rheumatic \& Musculoskeletal Medicine, University of Leeds, ${ }^{2}$ NIHR Leeds Biomedical Research Centre, Leeds Teaching Hospitals NHS Trust, Leeds, UK

Background: Tocilizumab (TCZ) has shown impressive outcomes in early RA (ERA) with clinical remission rates of up to $80 \% \%^{1}$. The speed and depth of remission of TCZ in treatment-naïve ERA have not been specifically evaluated.

Objectives: To evaluate the rate and depth of clinical and imaging response and remission, and timing of optimal response in ERA.

Methods: A prospective, open-label, RCT of active (DAS28 $\geq 3.2$ ), new-onset (symptom duration $\leq 12$ months) treatment-naive RA (2010 ACR/EULAR RA classification criteria), randomised 1:1, and treated with either TCZ $8 \mathrm{mg} / \mathrm{kg}$ (4-wkly) monotherapy or TCZ $8 \mathrm{mg} / \mathrm{kg}$ (4-wkly) and methotrexate (MTX) combination for 48 weeks.

Clinical response/remission rates, and patient reported outcomes (PRO) were evaluated at early (wks 4 and 12 ) timepoints, and wks 24 and 48 . High resolution US dominant hand ( \pm other baseline active joints) was performed at baseline, wks 12, 24 and 48. Odds ratios were calculated using Firth logistic regression.

Results: 20 pt [16 female; 13 RF+, 15 ACPA+; mean(SD) age 55.25(12) years] were recruited; baseline mean(SD) DAS28-ESR 5.98(1.21), HAQ 1.64(0.67). High-hurdle endpoints: at wk4, 30\%, 95\% and 35\% achieved DAS28-ESR rem, EULAR and ACR50 response respectively; and continued to increase, peaking at wk48 with $67 \%, 93 \%$ and $63 \%$ respectively [OR wk4 1.0, 0.3, 0.7 and wk48 1.4, 3.0, 4.0 respectively]. Sustained DAS28-ESR remission (8 successive weeks) was observed in $40 \%$; chisq $=0.5, p=0.462$; mean $(90 \% \mathrm{Cl})$ time to remission 38.3 (33.2, 43.3) wks

PRO: Baseline median(IQR) VASGH 56.5(29,71.5) improved by wk4 to 24 $(14,54)$, maintained wk48 24.5(3.5,46.5); VASDA from 62(54,77.5) to wk4 22 $(12.5,49.5)$, further improved by wk48 14.5(3.5,44.5); VASPain from 63.5(42,77) to wk4 19.5(4,53), maintained wk48 17.5(2.5,49.5). Similarly, median(IQR) HAQ at baseline $1.63(1.25,2.13)$ decreased by wk4 1.13(0.19,2.0) and wk48 0.75 (0.19,1.38).

Ultrasound: Baseline mean grey scale (GS) and power Doppler (PD) (as well as DAS28-ESR) are shown in the figure below, illustrating rapid reduction by wk4 that continued through the study period to all achieving $P D=0$ by wk48. Baseline median $(I Q R)$ erosions $0(0,0)$ remained unchanged throughout study. Baseline GS and PD appeared to associate with achievement of DAS44ESR remission $(p=0.038$ and $p=0.043$ respectively).

No meaningful numerical differences between the two treatment arms was recorded.

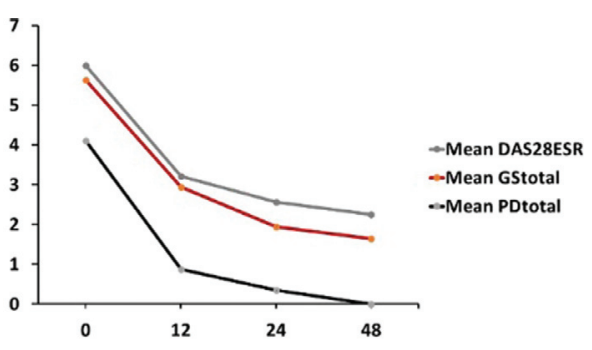

Conclusions: Conclusion TCZ in ERA (both monotherapy and TCZ/MTX combination) was associated with rapid clinical and imaging improvements, strikingly abolishing PD; with sustained remission in almost half the patients. The peak imaging response was noted at 48 weeks. Impressively, rapid PRO improvement

was also observed. These data indicate convincing patient-relevant, imagingdetermined depth of remission in a new-onset, treatment-naïve RA cohort.

\title{
REFERENCE:
}

[1] Bijlsma JWJ, et al. Lancet 2016; 388:10042;343-355.

Acknowledgements: This was an investigator-initiated study supported by Roche pharmaceuticals.

Disclosure of Interest: L. Hunt: None declared, E. Hensor: None declared, K. Naraghi: None declared, R. Wakefield: None declared, P. Emery Grant/research support from: Abbvie, AstraZeneca, Pfizer Ltd, Roche, Consultant for: Abbvie, AstraZeneca, BMS, Eli Lilly, MSD, Pfizer, Roche, Sandoz, M. Buch Grant/ research support from: Pfizer Ltd, Roche, Consultant for: Abbvie, BMS, Eli Lilly, Pfizer, Roche, Sandoz

DOI: 10.1136/annrheumdis-2018-eular.5494

\section{SAT0189 DYNAMICS OF CIRCULATING TNF DURING ADALIMUMAB TREATMENT OF RHEUMATOID ARTHRITIS USING A NOVEL DRUG-TOLERANT TNF ASSAY}

L. C. Berkhout ${ }^{1}$, M. J. I'Ami ${ }^{2}$, J. Ruwaard ${ }^{2}$, M. H. Hart ${ }^{1}$, P. Ooijevaar-de Heer ${ }^{1}$, K. Bloem ${ }^{1}$, M. T. Nurmohamed ${ }^{2,3}$, R. F. van Vollenhoven ${ }^{2,3,4}$, M. Boers $^{3,5}$, C. Smith ${ }^{6}$, G. Wolbink ${ }^{1,2}$, T. Rispens ${ }^{1} .{ }^{1}$ Department of Immunopathology, Sanquin Research, ${ }^{2}$ Amsterdam Rheumatology and immunology Center, Reade,

${ }^{3}$ Amsterdam Rheumatology and immunology Center, VU University Medical Center, ${ }^{4}$ Amsterdam Rheumatology and immunology Center, Academic Medical Center, ${ }^{5}$ Department of Epidemiology \& Biostatistics, VU University Medical Center, Amsterdam, Netherlands, ${ }^{6}$ St. John's Institute of Dermatology, Division of Genetics and Molecular Medicine, Kings College London, London, UK

Background: Tumor necrosis factor- (TNF) inhibitors are effective in the treatment of rheumatoid arthritis (RA); these include adalimumab, which binds TNF to form inactive complexes. Once in remission, a proportion of patients can successfully discontinue adalimumab treatment, indicating that blocking TNF is no longer necessary for disease control. We developed a novel assay that can quantify TNF in the presence of large amounts of TNF-inhibitor, i.e. a 'drug-tolerant' assay.

Objectives: To investigate, for the first time, the relationship between TNF levels and disease course during adalimumab treatment.

Methods: The new drug-tolerant competition enzyme-linked immunosorbent (ELISA) assay was used to quantify TNF levels on initiation and during 2 years of adalimumab treatment in 206 consecutive RA patients. The relationship between TNF levels and clinical response was evaluated.

Results: Circulating TNF levels were close to the detection limit at baseline, but TNF levels increased on average $>50$-fold upon adalimumab treatment (figure 1A; black lines show median (IQR)), and reached a stable level in time in the majority of patients (figure 1B; representatives of $n=206$ ), regardless of disease activity. During treatment, TNF was in complex with adalimumab, and recovered as inactive 3:1 adalimumab:TNF complexes. Low TNF levels at week four were associated with a higher frequency of anti-drug antibodies (ADAs) at subsequent time points (figure 1C) and significantly less methotrexate (MTX) use at baseline. Furthermore, week four TNF levels were significantly correlated with SDAI score, with significantly lower TNF levels in patients who did not reach remission (Spearman $r=-0.18 ; p=0.015$; figure 1D)

A

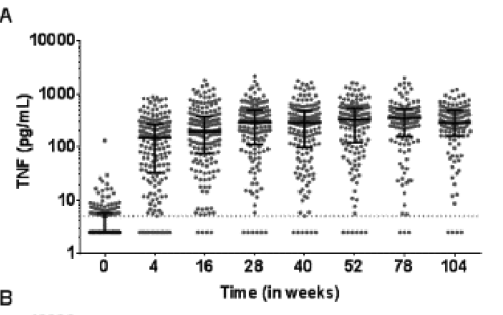

(100

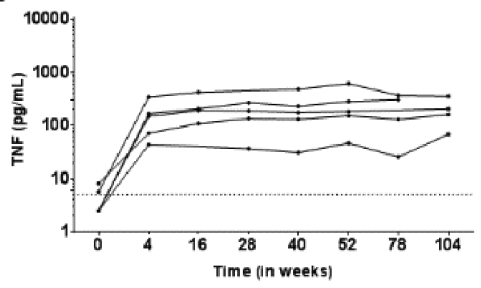

C
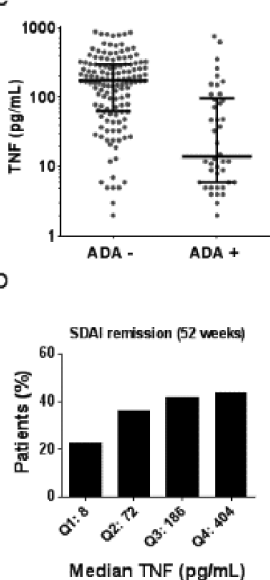

Conclusions: TNF levels, mostly in complexed form, do not appear to decline in patients that reach remission, and may therefore not be predictive for treatment 
discontinuation. However, low complexed TNF levels in the early phase of treatment (wk 4) are strongly associated with ADA formation and can be used to identify non-responders in the early phase of treatment.

Disclosure of Interest: L. Berkhout: None declared, M. I'Ami: None declared, J. Ruwaard: None declared, M. Hart: None declared, P. Ooijevaar-de Heer: None declared, K. Bloem: None declared, M. Nurmohamed Consultant for: Abbott, Roche, Pfizer, MSD, UCB, SOBI, BMS, Speakers bureau: Abbott, Roche, Pfizer, R. van Vollenhoven Grant/research support from: AbbVie, BMS, GSK, Pfizer, UCB, Consultant for: AbbVie, AstraZeneca, Biotest, BMS, Celgene, GSK, Janssen, Lilly, Novartis, Pfizer, UCB, M. Boers: None declared, C. Smith: None declared, G. Wolbink Grant/research support from: Pfizer, Speakers bureau: Pfizer, UCB, AbbVie, Biogen, BMS, T. Rispens Grant/research support from: Genmab, Speakers bureau: Pfizer, AbbVie, Regeneron

DOI: 10.1136/annrheumdis-2018-eular.3749

\section{SAT0190 RISK FOR OPPORTUNISTIC INFECTIONS IN RHEUMATOID ARTHRITIS TREATED WITH BDMARDS IN CLINICAL PRACTICE, 10 YEARS OF FOLLOW UP}

L. Leon ${ }^{1,2, *}$, M. Peñuela ${ }^{3}$, F. J. Candel ${ }^{3}$, D. Freites ${ }^{1}$, L. Rodriguez-Rodriguez ${ }^{1}$, L. Abasolo ${ }^{1}$. ${ }^{1}$ Rheumatology and Idissc, Hospital Clinico San Carlos, ${ }^{2}$ Health Sciences, Universidad Camilo Jose Cela, ${ }^{3}$ Microbiology, Hospital Clinico San Carlos, Madrid, Spain

Background: Biologic disease-modifying anti-rheumatic drugs (bDMARDs) may be associated with opportunistic infections (OI).

Objectives: Our purposes were to describe the incidence of $\mathrm{Ol}$ in Rheumatoid Arthritis (RA) taking bDMARDs, and compare the risk of OI between TNF-targeted and non-TNF-targeted biologics.

Methods: Retrospective longitudinal observational study from 2007 to 2017 . We included RA patients, diagnosed and followed in our outpatient clinic, whom started treatment with a TNF-targeted bDMARD [etanercept (ETN), golimumab (GOLI), certolizumab (CTZ), infliximab (IFX), adalimumab (ADA)], or non-TNF-targeted bDMARD [rituximab (RTX), abatacept (ABA), or tocilizumab (TCZ)]. According to microbiologist criteria, we consider OI when there was a positive culture (virus, fungus, bacterial, parasitary) or compatible symptoms that respond to specific treatment. The independent variable was the Type of targeted bDMARD: TNF-targeted vs non-TNF-targeted. Secondary variables: sociodemographic; clinical and other therapies. We used survival techniques to estimate the incidence of Ol, expressed per 1000 patient-year [CI $95 \%$ ]. The exposure time was defined from the start date of each bDMARD to the development of an OI, loss of follow up or end of study (01/02/2017). We performed Cox multivariate regression models to compare the risk of Ol between the types of bDMARD used. Results were expressed in Hazard ratio (HR).

Results: 441 RA patients were included, starting 761 different courses of bDMARD treatment. $81 \%$ were women with a mean age at first bDMARD of 57.3 \pm 14 years. The median time from onset of bDMARD until onset of OI was 3.1 years [0.5-4.6]. More than $90 \%$ of patients were on steroids. $71.3 \%$ of the courses were TNF-targeted bDMARD (ADA 39\%, ETN 33\%, CTZ 15\%, IFX 9\%, GOLI $2 \%$ ), and $28.7 \%$ non-TNF-targeted bDMARD (RTX 60\%, ABA 21\%, TCZ 18\%). There were 38 OI [26 Virus (18 Herpes Zoster, 2 virus B reactivation, 3 virus C reactivation, 1 Epstein Barr, 1 Avian flu, 1 CMV), 6 Fungus (5 Candida, 1 Trichophyton), 5 Bacterian (1 Legionella, 1 Salmonella, 3 TB)] and 1 parasitary (Leishmania)]. 9 of them required hospitalization and one died (Candida). The global incidence of OI was 23.1 [16.8-31.8]. TNF-targeted bDMARD had 26 OI, with an incidence of 20.8 [14.1-30.6], and non-TNF-targeted bDMARD with 12 Ol, incidence 30.4 [17.3-53.6]. We not find statistical differences in the rate of OI between TNF-targeted vs non-TNF-targeted in the multivariate model, adjusted by age, sex and calendar-time (HR 1.37, $\mathrm{p}=0.4)$. Male sex was found a predictor of $\mathrm{OI}$ in the multivariate analysis (HR 2.17, $\mathrm{p}=0.04)$. Age (HR 1.02, $\mathrm{p}=0.08)$, concomitant treatment with corticosteroids (HR 6.61, $\mathrm{p}=0.05$ ) and leukopenia (HR 2.7, $\mathrm{p}=0.08$ ) showed a tendency to increase the risk of $O \mathrm{I}$.

Conclusions: Incidence of Ol due to bDMARDs was near 23 cases per 1000 patients-year. Crude incidence was higher for non-TNF-targeted bDMARD compared to TNF-targeted bDMARD. Nevertheless this difference was not maintained in the multivariate model, reflecting that many of the variability in patients' risk of Ol development were driven by factors other than biological agent exposure. Close monitoring should be taken in those RA patients taking bDMARD, corticoids, and with leukopenia.

Disclosure of Interest: None declared

DOI: 10.1136/annrheumdis-2018-eular.5424

\section{SAT0191 THE PATIENT PERSPECTIVE ON BDMARD DOSE REDUCTION: A MIXED METHODS STUDY}

L. M. Verhoef ${ }^{1}$, E. M. Selten ${ }^{1}$, J. E. Vriezekolk ${ }^{1}$, A. J. de Jong ${ }^{2}$, F. H. van den Hoogen ${ }^{1,3}$, A. A. den Broeder ${ }^{1,3}$, M. E. Hulscher ${ }^{4} .{ }^{1}$ Rheumatology, Sint Maartenskliniek, Nijmegen, ${ }^{2}$ Rheumatology, Rijnstate, Arnhem, ${ }^{3}$ Rheumatology,

${ }^{4}$ IQ healthcare, RIHS, Radboudumc, Nijmegen, Netherlands

Background: Dose reduction of bDMARDs, after low disease activity is reached, is safe and effective.(1) To date, few studies have focused on bDMARD dose reduction from the patient perspective.

Objectives: The aim of this study was to identify the factors that play a role for patients with RA when considering bDMARD dose reduction, and to determine their relative importance.

Methods: A mixed methods design was used in which we 1) identified influenc ing factors with interviews and 2) ranked these factors using a Maximum Difference Scaling (MaxDiff) survey.

Sub study 1: We performed semi-structured interviews with 22 RA patients. Interviews were transcribed verbatim and two researchers analyzed the transcriptions by inductive thematic analysis.

Sub study 2: The influencing factors were derived from the interviews and used in a MaxDiff survey with RA patients from 3 different centers in the Netherlands ( $N=192$; an academic hospital, a specialized hospital and a large general hospital). Besides questions about patient characteristics, the survey included $18 \mathrm{Max}$ Diff questions in which patients were asked to choose the most and least important factor from a subset of 5 factors. A relative importance score for each factor was calculated using hierarchical Bayes modeling.

Results: Thirty factors were identified from the interviews and used in the survey. Most respondents had a positive attitude towards bDMARD dose reduction (table 1). The top-10 of factors (table 2) shows that patients are concerned that dose reduction will lead to a disease flare that affects their daily life (pain, function). It is important for them to know that it is possible to increase the dose if (further) reduction is not possible and that the bDMARD will be effective again. Patients value the opinion of their rheumatologist, and being involved in the decision to start tapering is highly ranked as well.

Table 1 Patient characteristics (mean (sd) or $\mathrm{n}(\%)$ )

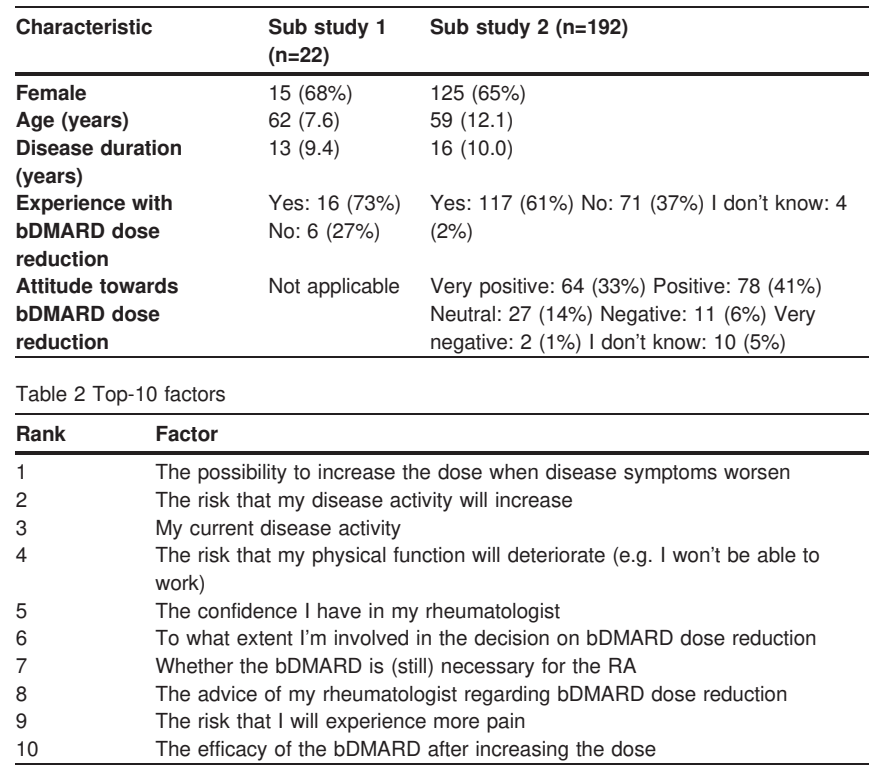

Conclusions: The results from this study could facilitate implementation of bDMARD dose reduction by informing care providers on what is important for patients and providing a basis for shared decision making.

\section{REFERENCE:}

[1] Nam JL, et al. Ann Rheum Dis 2017 Jun;76(6):1113-1136.

Disclosure of Interest: None declared DOI: 10.1136/annrheumdis-2018-eular.2526 